\title{
Система поддержки принятия решений по управлению рисками опасных ситуаиий в сложных системах газоснабжения
}

\author{
P.P. Кантюков ${ }^{1}$, к.т.н., доцент, зам. генерального директора по науке, \\ R_Kantyukov@vniigaz.gazprom.ru \\ O.Б. Бутуссов 2,3, д.фp.-M.н., npoфeccop, butusov-1@mail.ru
}

В.П. Мешалкин ${ }^{2,4}$, д.т.н., академик РАН, профессор, зав. кафедрой, vpmeshalkin@gmail.com

B.M. Панарин 5, д.m.н., профессор, зав.кафедрой, panarin-tsu@yandex.ru

${ }^{1}$ НИИ природных газов и газовых технологий - Газпром ВНИИГАЗ, пос. Развилка, 142717, Россия

2 Российский химико-технологический университет им. Д.И. Менделеева, 2. Москва, 125047, Россия

3 Российский государственный соииальный университет, г. Москва, 129226, Россия

${ }^{4}$ Институт общей и неорганической химии им. Н.С. Курнакова РАН, 2. Москва, 119991, Россия

5 Тульский государственный университет, г. Тула, 300012, Россия

Разработаны архитектура и программно-информационное обеспечение системы поддержки принятия решений по анализу и снижению рисков аварийных ситуаций в сложных системах газоснабжения и по уменьшению воздействия аварийных ситуаций на лесные массивы.

Для выработки рациональных решений в системах поддержки принятия решений используются специальные блоки расчета показателей технологических и экологических рисков. Расчет показателей экологических рисков осуществляется по оценкам воздействия на лесные массивы аварийных ситуаций в сложных системах газоснабжения. При разработке ПО системы поддержки принятия решений проведена детальная классификация возможных аварийных ситуаций в сложных системах газоснабжения и разработаны процедуры принятия соответствующих научно-обоснованных решений системы поддержки принятия решений по ликвидации опасных ситуаций. При разработке алгоритмического и программно-информационного обеспечения системы впервые введен ряд новых показателей безопасности сложных систем газоснабжения.

В качестве основных показателей рисков использованы техногенные риски (промышленный, экологический, социальный) возникновения опасных (аварийных) ситуаций на сложных системах газоснабжения. Риски позволяют оценить возможный ущерб природной среде в случае возникновения аварийных ситуаций в сложных системах газоснабжения. Для оценки экологического ущерба, нанесенного лесным массивам аварийными ситуациями, на сложных системах газоснабжения предложено использовать сегментацию спутниковых изображений территории с помощью методов кластерного анализа. При этом компьютерное моделирование атмосферного переноса газовых выбросов, биогеоценотические БД и зависимости «доза-эффект» позволяют непосредственно определять интегральные показатели ущерба лесным массивам по величине дозы.

При разработке ПО использованы Microsoft Visual Studio (языки программирования Си++ и Сишарп), пакет компьютерной математики Матлаб, а также БД и современные средства информационнокомпьютерных технологий.

Ключевые слова: система поддержки принятия решений, программно-информационное обеспечение, сложные системы газоснабжения, лесные массивы, аварийные и опасные ситуации, техногенные и экологические риски, эколого-экономическая оптимизация, зависимость «доза-эффект».

Одной из важных задач функционирования сложных систем газоснабжения (ССГ) является минимизация рисков аварийных ситуаций. Для решения этой задачи при эксплуатации ССГ необходимо учитывать и прогнозировать возможное возникновение и развитие опасных и чрезвычайных ситуаций (ЧС) на объектах ССГ [1-4]. В результате проведенных исследований по анализу и классификации опасных ситуаций в ССГ [5] разработано алгоритмическое 
обеспечение системь поддержки принятия решений (СППР) для прогнозирования и управления рисками опасных ситуаций в ССГ и принятия решений по уменьшению опасности возникновения ЧС. Архитектура и режимы функционирования СППР разработаны на основе методологии системного подхода в химической технологии, методов логистики ресурсосбережения и теории риска. Важнейшими компонентами алгоритмического и программно-информационного обеспечения СППР являются блоки оперативного анализа режимов функционирования объектов ССГ [5], реализующие три процедуры: предварительный качественный анализ опасностей, выявление последовательностей опасных ситуаций, прогнозирование последствий опасных ситуаций и расчет значений промышленного и экологического рисков.

Архитектура СППР представлена на рисунке 1. Основу архитектуры составляют три группы модулей: группа модулей БД, группа модулей управления, оценки рисков и принятия решений, группа модулей анализа спутниковой информации. ПО разработано с помощью Microsoft Visual Studio и пакета компьютерной математики Матлаб.

\section{Алгоритмическое и программное} обеспечение СППР по расчету показателей надежности ССГ

Математическое обеспечение включает следующие алгоритмы обеспечения надежности

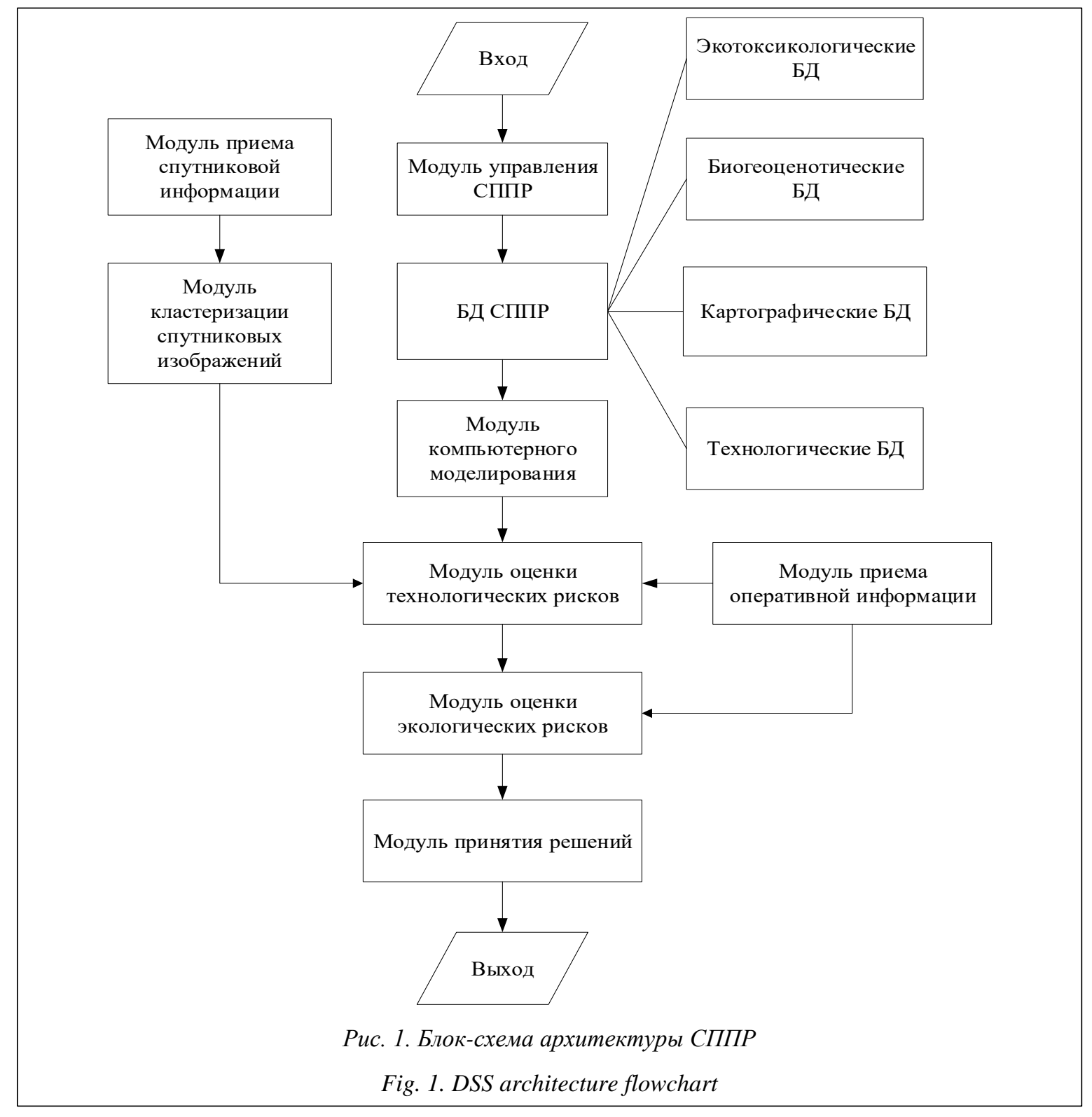


ССГ: алгоритмы обеспечения надежности технических коридоров магистральных газопроводов, входящих в структуру ССГ; алгоритмы расчета кольцевых структур ССГ для обеспечения бесперебойного снабжения потребителей; алгоритмы планирования, модернизации и оптимизации структуры трубопроводов ССГ на основе требуемых показателей надежности $[1,2]$.

ПО СППР состоит из укрупненных блоков (модулей) [1, 2].

1. Блок расчета показателей надежности проектируемых объектов ССГ, реализующий:

- выбор оптимальных технологических решений по управлению процессами транспорта газа;

- расчет основных инженерно-технических параметров ССГ;

- выбор направления трассы газопровода и ее месторасположения на территории;

- выбор материалов, типоразмеров оборудования и его изготовителей (марка стали, наличие и качество внешнего и внутреннего покрытия труб, конструкция и качество изготовления оборудования на компрессорных станциях, станциях охлаждения газа, газораспределительных станциия (ГРС) и других объектах газопровода);

- выбор системы электрохимической заųuты (ЭХЗ) для ССГ в системе ЭХЗ

2. Блок выбора рациональных схемноструктурных конструкций ССГ, а именно:

- технологических схем объектов (компрессорных и газораспределительных станций);

- вида сопряжения проектируемого нового объекта с действующей ССГ для маневрирования потоками, управления запасами газа;

$\mathrm{CC \Gamma}$;

- вида резервирования оборудования

- видов обеспечения резервов производственной мощности и их распределения по объектам ССГ;

- средств автоматизации и информатизации проектируемой ССГ по видам диагностического оборудования и измерительной аппаратуры;

- видов диагностического обслуживания объектов ССГ.

3. Блок выбора типа конструкций оборудования ССГ, стратегии организации технического обслуживания и ремонтов оборудования.

4. Блок выбора конструкции подземных хранилищ газа и их размещения.
5. Блок определения оптимальных резервов производственной мощности газопроводов и резервов производственных мощностей многониточного коридора для определения режимов транспортировки, хранения и распределения газа в ССГ.

В архитектуру разработанной СППР входит блок, программно реализующий алгоритм расчета показателей безопасности ССГ по представительной статистической информации и алгоритмы обеспечения показателей безопасности при проектировании, организации строительства и эксплуатации энергоресурсоэффективных развивающихся ССГ.

В архитектуру СППР также входят блоки, реализующие алгоритмы принятия решений по обеспечению безопасности действующих систем газоснабжения $[1,2]$, а именно:

- алгоритм сопоставления (ранжирования) отдельных объектов ССГ на основе использования интегральных показателей техногенного риска;

- алгоритм планирования стратегии технического обслуживания и предупредительных ремонтов оборудования (ежегодное и среднесрочное), распределения средств на ремонты между объектами или подразделениями одного предприятия как при эксплуатации, так и при реконструкции ССГ;

- алгоритмы расчета эксплуатационных затрат, а также сумм технологического и экологического страхования на основе использования показателей промышленного и экологического рисков;

- алгоритмы разработки научно-обоснованных организационно-технических мероприятий по повышению безопасности ССГ на основе использования показателей риска;

- алгоритмы выявления наиболее опасных узких мест ССГ и их учета при разработке мероприятий по техническому и экологическому мониторингу ССГ.

\section{Алгоритмическое обеспечение методов оценки воздействия ССГ на лесные массивы}

В архитектуру разработанной СППР входит алгоритмическое и программное обеспечение методов оценки воздействия ССГ на лесные массивы (ЛМ) [6-8]. Разработанные алгоритмы оценки основаны на теории «доза-эффект»-зависимостей применительно к ЛМ [9]. В теории «доза-эффект»-зависимостей к параметрам эффекта относятся экстенсивные (запас 
древесины, наземная фитомасса) и структурные (видовое разнообразие) характеристики ЛМ. К параметрам дозы относятся показатели техногенного воздействия. Одним из важных показателей эффекта для ЛМ является видовое разнообразие. Этот показатель может быть использован как интегральный индекс для оценки экологического ущерба, нанесенного техногенными воздействиями на ЛМ объектов ССГ. Оценка видового разнообразия рассчитывается по формуле

$$
E_{C}=-\sum_{i=1}^{I} \log \left(N_{i} / N\right),
$$

где $E_{c}$ - экологическая ценность ЛМ; $N_{i}$ - число объектов $i$-го класса; $N$ - общее число объектов в экосистеме ЛМ.

На рисунке 2 представлен двудольный информационный граф расчета зависимостей эколого-техногенного взаимодействия объектов ССГ и компонентов ЛМ в виде двудольного графа, а на рисунке 3 - блок-схема классифика-

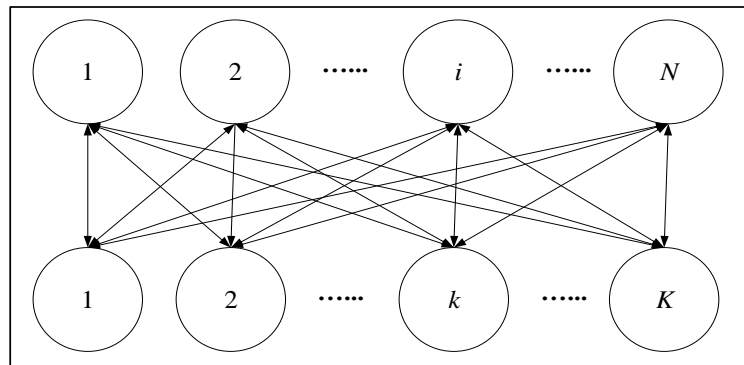

Рис. 2. Двудольный информационный граф эколого-техногенного взаимодействия объектов ССГ и ЛМ: і нумерует компоненты лесной экосистемы, $k$ нумерует объекты ССГ

Fig. 2. The bipartite information graph of the ecological and technogenic objects interaction: $i$ numbers the forest ecosystem components, $k$ numbers the technogenic objects

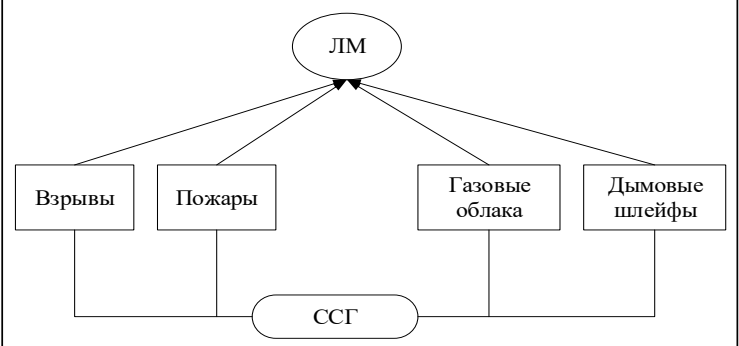

Рис. 3. Блок-схема классификации основных видов воздействия аварийных ситуаџий ССГ на ЛМ

Fig. 3. The classification flowchart of the technogenic impacts main types on forest stands ции основных видов воздействия аварийных ситуаций ССГ на состояние ЛМ [9].

Для расчета степени воздействия объектов ССГ на ЛМ в СППР использована система интегральных индексов экологического состояния ЛМ:

$$
\rho_{1 i}=\frac{\sum d_{i j}\left(\Delta B_{j}\right)_{F}}{\sum d_{i j} \Delta B_{j}} \frac{\sum d_{i j} B_{j}}{\sum d_{i j}\left(B_{j}\right)_{F}},
$$

где $\rho_{i}-$ интегральный индекс для $i$-го участка ЛМ; $B_{j}$ - измеряемые параметры экологического состояния ЛМ; $d_{i j}$ - численные коэффициенты разложения измеряемых параметров на внутреннем базисе; индекс $F$ - фоновое или максимальное значение параметра.

Для оценки воздействия на ЛМ объектов ССГ в СППР также используется блок, реализующий алгоритм относительных площадных оценок экологического ущерба:

$$
D=\frac{1}{S} \iint_{S}\left(1-\frac{1}{k} \sum_{k} E_{k}(x, y)\right) d S,
$$

где $S$ - площадь ЛМ; $E_{k}(x, y)=I_{k}(C(x, y))-$ «доза-эффект»-зависимость для $k$-го интегрального индекса; $C(x, y)$ - доза [9].

\section{Режимы функционирования СППР}

Представленная СППР функционирует в четырех режимах $[7,8]$.

1. Предварительный качественный анализ опасностей, способных превратиться в техногенные аварии и ЧС, для чего производится классификация инженерных объектов ССГ как техногенно-опасных систем. При обследовании инженерных объектов необходимо собрать и проанализировать достаточно большой объем технической информации.

2. Выявление и описание основных опасностей каждого из инженерных объектов. На этом этапе зафиксированные опасности «привязываются» к составным частям (элементам) объекта или операциям, к которым они имеют отношение.

3. Отбор происшествий (событий). Отбор происшествий завершают составлением сценариев развития аварий, отобранных для последующего анализа риска. Сценарии представляют описания развития последовательности событий от потенциальных опасностей до реализации инцидента.

4. Анализ последствий опасных ситуаций на основе: расчета количественных характеристик утечек природного газа, токсичных веществ и выделяемой энергии для каждого сценария развития аварии; расчета характеристик 
эмиссии токсичных веществ в окружающую среду; расчета распространения ударной волны или фронта пожара, которые ведут к человеческим жертвам; оценки воздействия на здоровье людей, повреждений материальных ценностей и ущерба лесным массивам; оценки техногенного риска объекта.

Для анализа последствий опасных ситуаций в СППР используются блок математического моделирования для моделирования различных инцидентов, например, разгерметизации оборудования, а также математические модели эмиссии химических веществ в окружающую среду.

В СППР программная реализация алгоритма расчета экологического риска осуществляется на основе оценки техногенных воздействий на ЛМ [9]. Экологический риск с учетом ущербов, нанесенных ЛМ, рассчитывается с помощью интегрального коэффициента сохранности ЛМ по следующей формуле:

$$
R=\iint_{G} \int_{0}^{C_{\max }}(1-I(x, y)) P\left(C_{0}(x, y)\right) d x d y d C_{0},
$$

где $I(x, y)$ - площадное распределение интегрального коэффициента сохранности; $P\left(C_{0}(x, y)\right)$ - плотность вероятности распределения по территории ЛМ воздействий аварий в ССГ с различными значениями начальных концентраций аварийных газов.
Для оценки пространственного распределения концентрации вредных веществ по территории ЛМ в СППР используется полуэмпирическое уравнение турбулентной диффузии. В инженерных расчетах для оценки скорости распространения аварийных облаков применяется стандартная методика РД 52.04.253-90, в которой учитывают три класса устойчивости атмосферы.

Системный анализ рисков завершается в СППР прогнозированием и расчетом оценок аварийно-техногенного риска.

\section{Заключение}

Оценка воздействия на ЛМ ССГ в виде техногенных рисков является сложной комплексной проблемой. Для ее решения разработана специальная СППР по управлению рисками опасных ситуаций в ССГ.

Для оценки экологического ущерба, нанесенного ЛМ аварийными ситуациями, на ССГ предложено использовать сегментацию спутниковых изображений территории с помощью методов кластерного анализа. При этом компьютерное моделирование атмосферного переноса газовых выбросов, биогеоценотические БД и зависимости «доза-эффект» позволяют непосредственно определять интегральные показатели ущерба ЛМ по величине дозы.

\section{Лumepamypa}

1. Кантюков Р.А., Кантюков Р.Р., Якупов Н.М., Тамеев И.М., Якупов С.Н. Проблемы безопасности трубопроводов // Газовая промышленность. 2012. № 6. С. 79-83.

2. Кантюков Р.А., Кантюков Р.Р., Хадиев М.Б., Хамидуллин И.В., Максимов В.А. Компрессоры в технологических процессах. Газораспределительные, компрессорные станции магистральных газопроводов и автомобильные газонаполнительные станции. Казань: Изд-во КФУ, 2014. 647 с.

3. Сухарев М.Г., Карасевич А.М. Модели надежности газоснабжающих систем // Автоматика и телемеханика. 2010. № 7. C. 149-159. DOI: 10.1134/S0005117910070155.

4. Сухарев М.Г., Карасевич А.М. Состояние и проблемы надежности единой и региональных систем газоснабжения // Изв. РАН. 2009. № 5. С. 34-40.

5. Сухарев М.Г., Калинина Э.В., Лапига А.Г. Статистический анализ аварийности газораспределительных систем // Территория НЕФТЕГАЗ. 2010. № 4. С. 16-19.

6. González H.A., De La Cruz J.M., De Andrés-Toro B., Risco-Martín J.L. Modeling and simulation of a gas distribution pipeline network. Applied Mathematical Modeling, 2009, vol. 33, iss. 3, pp. 1584-1600. DOI: 10.1016/j.apm.2008.02.012.

7. Ramachandra T.V., Krishna S.V., Shruthi B.V. Decision support system for regional domestic energy planning. J. of Sci. Ind. Res., 2005, vol. 64, pp. 163-174. URL: https://pdfs.semanticscholar.org/8242/ b159a43fe7cd4871c1f065cef94356314613.pdf (дата обращения: 15.03.2020).

8. Djebedjiana B., El-Naggara M., Shahin I. Optimal decision of gas distribution network: A case study, MEJ, 2011, vol. 36, no. 3, pp. 35-51.

9. Гриценко А.И., Акопова Г.С., Максимов В.М. Экология. Нефть и газ. М.: Наука, 1997. 598 с. 


\section{Decision support system for risk management of dangerous situations in complex gas supply systems}

R.R. Kantyukov ${ }^{1}$, Ph.D. (Engineering), Associate Professor, Deputy Director General for Science,

R_Kantyukov@vniigaz.gazprom.ru

O.B. Butusov ${ }^{2,3}$,Dr.Sc. (Physics and Mathematics), Professor, butusov-1@mail.ru

V.P. Meshalkin ${ }^{2,4}$, Dr.Sc. (Engineering), Academician of the RAS, Professor, Head of the Department, vpmeshalkin@gmail.com

V.M. Panarin ${ }^{5}$, Dr.Sc. (Engineering), Professor, Head of the Department, panarin-tsu@yandex.ru

${ }^{1}$ Scientific-Research Institute of Natural Gases and Gas Technologies - VNIIGAZ,

Razvilka vil., 142717, Russian Federation

${ }^{2}$ Dmitry Mendeleev University of Chemical Technology of Russia, Moscow, 125047, Russian Federation

${ }^{3}$ Russian State Social University, Moscow, 129226, Russian Federation

${ }^{4}$ Kurnakov Institute of General and Neorganic Chemistry of RAS, Moscow, 119991, Russian Federation

${ }^{5}$ Tula State University, Tula, 300012, Russian Federation

Abstract. The authors developed an architecture, software and decision support system (DSS) for analyzing and emergency risk reduction in complex gas supply systems (CGSS) and for reducing the impact on forest areas.

They use special modules for calculating technological and environmental risks in order to produce decisions in DSS. The ecological damage calculation is based on assessing the impact on the forest areas by the emergency situations in the CGSS. During the development of the DSS software, there was a detailed classification of possible emergency situations in the CGSS, as well as special procedures developed to produce appropriate system decisions for the dangerous situation control. When developing algorithmic and software for DSS, the authors introduced a number of new CGSS safety indicators for the first time.

Technogenic risks (industrial, environmental, social) of the dangerous (emergency) situations on the CGSS were taken as the main risk indicators. Risks make it possible to assess probable environmental damage in case of emergencies in the CGSS. Thepaper proposes to use segmentation of forest satellite images to assess the environmental damage caused by accidents in the CGSS. At the same time, computer modeling of atmospheric transport for gas emissions, biogeocenotic databases, and the "exposure-response" dependencies make it possible to determine the total damage to forests by the values of the exposure directly.

While developing DSS software, the authors used Microsoft Visual Studio (C++ and C-sharp programming languages), Matlab package, as well as databases and modern means of information and computer technologies.

Keywords: decision support system, software and information support, databases, complex gas supply systems, forests, emergency situations, industrial and environmental risks, exposure-response function.

\section{References}

1. Kantyukov R.A., Kantyukov R.R., Yakupov N.M., Tameev I.M., Yakupov S.N. Pipe safety problems. Gas Industry Magazine, 2012, no. 6, pp. 79-83 (in Russ.)

2. Kantyukov R.A., Kantyukov R.R., Khadiev M.B., Khamidullin I.V., Maksimov V.A. Compressors in Technological Processes. Gas Distribution, Compressor Stations of Gas Pipelines and Gas Filling Stations. Kazan, KSU Publ., 2014, 647 p. (in Russ.).

3. Sukharev M.G., Karasevich A.M. Reliability models for gas supply systems. Automation and Remote Control, 2010, no. 7, pp. 149-159. DOI: 10.1134/S0005117910070155 (in Russ.).

4. Sukharev M.G., Karasevich A.M. State of the art and problems of reliability of unified and regional gas supply systems. Bull. of the RAS, 2009, no. 5, pp. 34-40 (in Russ.).

5. Sukharev M.G., Kalinina E.V., Lapiga A.G. Statistical analysis of the accident rate of gas distribution systems. Territory of NEFTEGAZ, 2010, no. 4, pp. 16-19 (in Russ.).

6. González H.A., De La Cruz J.M., De Andrés-Toro B., Risco-Martín J.L. Modeling and simulation of a gas distribution pipeline network. Applied Mathematical Modeling, 2009, vol. 33, iss. 3, pp. 1584-1600. DOI: 10.1016/j.apm.2008.02.012.

7. Ramachandra T.V., Krishna S.V., Shruthi B.V. Decision support system for regional domestic energy planning. J. of Sci. Ind. Res., 2005, vol. 64, pp. 163-174. Available at: https://pdfs.semanticscholar.org/8242/ b159a43fe7cd4871c1f065cef94356314613.pdf (accessed March 15, 2020). 
8. Djebedjiana B., El-Naggara M., Shahin I. Optimal decision of gas distribution network: A case study. MEJ, 2011, vol. 36, no. 3, pp. 35-51.

9. Gritsenko A.I., Akopova G.S., Maksimov V.M. Ecology. Oil and Gas. Moscow, Nauka, 1997, 598 p.

\section{Для цитирования}

Кантюков Р.Р., Бутусов О.Б., Мешалкин В.П., Панарин В.М. Система поддержки принятия решений по управлению рисками опасных ситуаций в сложных системах газоснабжения // Программные продукты и системы. 2020. Т. 33. № 2. С. 250-256. DOI: 10.15827/0236-235X. $130.250-256$.

\section{For citation}

Kantyukov R.R., Butusov O.B., Meshalkin V.P., Panarin V.M. Decision support system for risk management of dangerous situations in complex gas supply systems. Software \& Systems, 2020, vol. 33, no. 2, pp. 250-256 (in Russ.). DOI: 10.15827/0236-235X.130.250-256. 